\title{
ARTICLE OPEN The effects of altered BMP4 signaling in first branchial- arch-derived murine embryonic orofacial tissues
}

\author{
Jue $\mathrm{Xu}^{1}$, Meiling Chen ${ }^{2}$, Yanan $\mathrm{Yan}^{2}$, Qiaoxue Zhao ${ }^{2}$, Meiying Shao ${ }^{\boxplus 凶}$ and Zhen Huang $\mathbb{D}^{2 凶}$
}

The first branchial arch (BA1), which is derived from cranial neural crest (CNC) cells, gives rise to various orofacial tissues. Cre mice are widely used for the determination of CNC and exploration of gene functions in orofacial development. However, there is a lack of Cre mice specifically marked BA1's cells. Pax2-Cre allele was previously generated and has been widely used in the field of inner ear development. Here, by compounding Pax2-Cre and R26R-mTmG mice, we found a specific expression pattern of Pax ${ }^{+}$cells that marked BA1's mesenchymal cells and the BA1-derivatives. Compared to Pax2-Cre; R26R-mTmG allele, GFP ${ }^{+}$cells were abundantly found both in BA1 and second branchial arch in Wnt1-Cre;R26R-mTmG mice. As BMP4 signaling is required for orofacial development, we over-activated Bmp4 by using Pax2-Cre; pMes-BMP4 strain. Interestingly, our results showed bilateral hyperplasia between the upper and lower teeth. We also compare the phenotypes of Wnt1-Cre; pMes-BMP4 and Pax2-Cre; pMesBMP4 strains and found severe deformation of molar buds, palate, and maxilla-mandibular bony structures in Wnt1-Cre; pMesBMP4 mice; however, the morphology of these orofacial organs were comparable between controls and Pax2-Cre; $p M e s-B M P 4$ mice except for bilateral hyperplastic tissues. We further explore the properties of the hyperplastic tissue and found it is not derived from Run $\times 2^{+}$cells but expresses Msx1, and probably caused by abnormal cell proliferation and altered expression pattern of p-Smad1/5/8. In sum, our findings suggest altering BMP4 signaling in BA1-specific cell lineage may lead to unique phenotypes in orofacial regions, further hinting that Pax2-Cre mice could be a new model for genetic manipulation of BA1-derived organogenesis in the orofacial region.

International Journal of Oral Science (2021)13:40

; https://doi.org/10.1038/s41368-021-00142-4

\section{INTRODUCTION}

The normal embryogenesis and morphogenesis of cranial-facial organs such as frontonasal, midbrain, craniofacial skeleton, eyes, nose, lip, palate, jaw, teeth, and external and inner ears depend on the appropriate migration and differentiation of cranial neural crest (CNC) cells ${ }^{1,2}$. During cranial-facial development, CNC cells migrate to their ventral-lateral destination and form branchial arches (BAs). Specifically, the first branchial arch (BA1) is further divided into maxillary and mandibular prominences and is essential for the morphogenesis of tissues in maxillamandibular regions. Consequently, BA1 differentiated into characteristic orofacial tissues, such as teeth, dentary bone, and secondary palate . $^{3,4}$.

Cre mice are widely used in inducible systems for studying the functions of a specific gene. In the field of orofacial organogenesis, transgenic mice were used to trace the lineage-dependent CNC cells. Wnt1-Cre and PO-Cre are extensively used in the study of craniofacial development ${ }^{5-8}$. Specifically, as Wnt1 promoter activated in neural crest pre- and post-migratory progenitors, Wnt1-Cre mice were accepted as the most widely used allele in studying the patterning of craniofacial organs $s^{5,9,10}$. However, the $\mathrm{Wnt}^{+}$or $\mathrm{PO}^{+}$cells existed not only in $\mathrm{BA} 1$ but also widely distributed in the second $B A(B A 2)^{10,11}$. Although some other specific Cre mice are also used for studying the development of orofacial organs or tissues, they are restricted to a certain subgroup of CNC cells. Osr2-Cre mice were generated and used for tissue-specific studies in palate development ${ }^{11-13}$. But the Osr2-Cre is limited to study the palatogenesis and pattern formation during palate development because of the specific expression of Osr2 in palatal shelves. Dmp1-Cre is also an allele that can be used in the craniofacial region; however, the $D m p 1^{+}$ cells were restricted in osteocytes in maxilla-mandibular regions ${ }^{14,15}$. Osx-Cre mice is another strain that has been used for studying the mechanisms of both bone and dental mesenchymal tissue ${ }^{16,17}$. Therefore, it is difficult to study the specific BA1derived orofacial organs or tissues by using the above Cre mice.

The bone morphogenetic proteins (BMPs) are morphogens that are essential for pattern formation of neurogenesis, ossification, organogenesis, and the pre- and post-natal tissue growth ${ }^{18}$. In orofacial regions, BMP signaling is necessary for organs or tissues' development including palate, teeth, skeletons, and temporomandibular joint (TMJ) ${ }^{19}$. BMPs, BMP receptor la (BMPRla), and BMP antagonist are expressed in the palate and regulate palatogenesis of mice ${ }^{20-24}$. In teeth development, BMP signaling plays critical roles in initiation, morphogenesis, mineralization, and root formation ${ }^{19,25}$. Lack or over-activation of BMP signaling in either tooth's epithelial or mesenchymal cells leads to malformation of tooth ${ }^{21,26-28}$. The skeletogenesis in cranial-facial including the skull formation as well as mandibular development are regulated by BMP signaling ${ }^{29-32}$. Members of BMP signaling such

\footnotetext{
${ }^{1}$ West China School of Public Health and Department of Stomatology, West China Fourth Hospital, Sichuan University, Chengdu, China and ${ }^{2}$ Southern Center for Biomedical Research and Fujian Key Laboratory of Developmental and Neuro Biology, College of Life Sciences, Fujian Normal University, Fuzhou, China

Correspondence: Meiying Shao (shaomeiying2013@163.com) or Zhen Huang (zhuang@fjnu.edu.cn)
}

Received: 25 January 2021 Revised: 31 August 2021 Accepted: 5 September 2021

Published online: 29 November 2021 
as Bmprla and Bmp2 also exert biological functions during the development of $\mathrm{TMJ}^{33,34}$. Among these, Bmp4 expresses in maxillary and mandibular processes of $\mathrm{BA} 1$ and contributes to the development of orofacial organs such as tooth, palate, maxilla, and mandible ${ }^{19}$. However, the researchers all selected the abovementioned traditional Cre mice (Wnt1-Cre etc.) to explore the mechanisms of BMP4 signaling in the orofacial region $21,27,32,35$. According to the limitations of the Cre distribution of these mouse strains, the exact influences of BMP4 signaling in orofacial organs or tissues may probably be hidden.

In this study, we took advantage of Pax2-Cre ${ }^{36}$ and $R 26 R$ $\mathrm{mTmG} \mathrm{mice}{ }^{37}$ to trace the lineage of $\mathrm{Pax2}^{+}$cells in the oral-facial region and found these cells were specifically expressed in BA1 while the $\mathrm{Wnt}^{+}$cells were both detected in BA1 and BA2. Thus, the Pax2-Cre had immense potential as a transgenic mouse model for the lineage tracing of BA1-derived mesenchymal cells. Subsequently, we use Pax2-Cre mice to detect the function of BMP4 signaling in BA1-derived orofacial mesenchyme by using pMes-Bmp4 allele ${ }^{32}$. We also compared the craniofacial phenotype of Pax2-Cre; pMes-BMP4 and Wnt1-Cre; pMes-BMP4 mice. Our results reveal the specific effects of BMP4 signaling in BA1derived murine embryonic orofacial regions.

\section{RESULTS}

$\mathrm{Pax}^{+}$cell lineage was specifically expressed in BA1 and the derivatives

Ohyama and colleagues have generated Pax2-Cre mice and further successfully used Pax2-Cre mice in their studies focused on the development of inner ear $^{36,38}$. Here we first compounded Pax2-Cre and R26R-mTmG mice to determine the expression of Pax2 and to trace $\mathrm{Pax}^{+}$lineage cells in the developing orofacial regions.

Live imaging on Pax2-Cre; R26R-mTmG embryos revealed $\mathrm{Pax}^{+}$ lineage cells (labeled as $\mathrm{GFP}^{+}$cells) existed specifically in BA1 except for the head region (Fig. 1a, b) while the GFP ${ }^{+}$cells of Wnt1-Cre; R26R-mTmG mice were found not only in midbrain but also in BA1 and BA2 from E10.5 through E11.5 (Fig. 1a', b). An intensive distribution of $\mathrm{Pax}^{+}$and $\mathrm{Wnt}^{+}$cell lineages in the BAs was observed by anti-GFP staining at E10.5 (Fig. 1C, C). GFP- positive cells were limited in mesenchyme of BA1 and barely found in BA2 in Pax2-Cre; R26R-mTmG mice (Fig. 1c). On the other hand, $W_{n t} 1^{+}$lineage cells were found both in BA1 and BA2 mesenchyme (Fig. 1C). The Pax2 ${ }^{+}$and $\mathrm{Wnt}^{+}$cells were detected in mesenchyme of orofacial organs (Fig. 1d, d).

Forced Bmp4 in BA1-derived orofacial regions leads to hyperplasia between upper and lower jaws

To evaluate the effects of BMP4 signaling in BA1-derived orofacial regions, we used Pax2-Cre allele to locally activate Bmp4 by compounding the Pax2-Cre allele with pMes-BMP4 allele.

We found the Pax2-Cre; pMes-BMP4 mice died soon after birth, and compared to the control, the stomach of the mutant lack of milk (data not shown). But the mutant mice did not exhibit cleft palate defect, which is one of the most common birth defect of mice. Instead, the oral view of the whole-mount unraveled a unique palatal abnormity: the bilateral soft tissue hyperplasia located between upper and lower jaws (Fig. 2b, marked by asterisk). Histological analysis of Pax2-Cre; pMes-BMP4 mice showed the existence of hyperplastic soft tissue as early as E13.5 (Fig. 2c). Specifically, we found the abnormal tissue extended from the region between maxillary and mandibular molars and consists of epithelial and mesenchymal cells. In addition, the size of the hyperplastic soft tissue gradually increased from E13.5 to E15.5, and then decreased after E15.5 (Fig. 2 $c^{\prime}-g$ ). Although the growth direction of tooth buds was changed because of the hyperplasia, the size morphology was comparable to the controls (Fig. 2h, i). Although the morphology of tongue in mutants seem different from the controls, the MF20 marked muscle were comparable to the controls (Supplemental Fig. 1).

Wnt1-Cre; pMes-BMP4 and Pax2-Cre; pMes-BMP4 mutant mice exhibit different phenotypes in orofacial organs

To give a clear picture of orofacial bone and tooth phenotypes in CNC-derived cells, we compare the tooth buds, mandibular bones, Meckel's cartilage, and palate between Wnt1Cre; pMes-Bmp4 and Pax2-Cre; pMes-BMP4 heads (Fig. 3). In our results, the molar buds, mandibular bony structures, and Meckel's cartilage were severely destructed in Wnt1Cre; pMes-Bmp4 mice (Fig. 3b); however, the
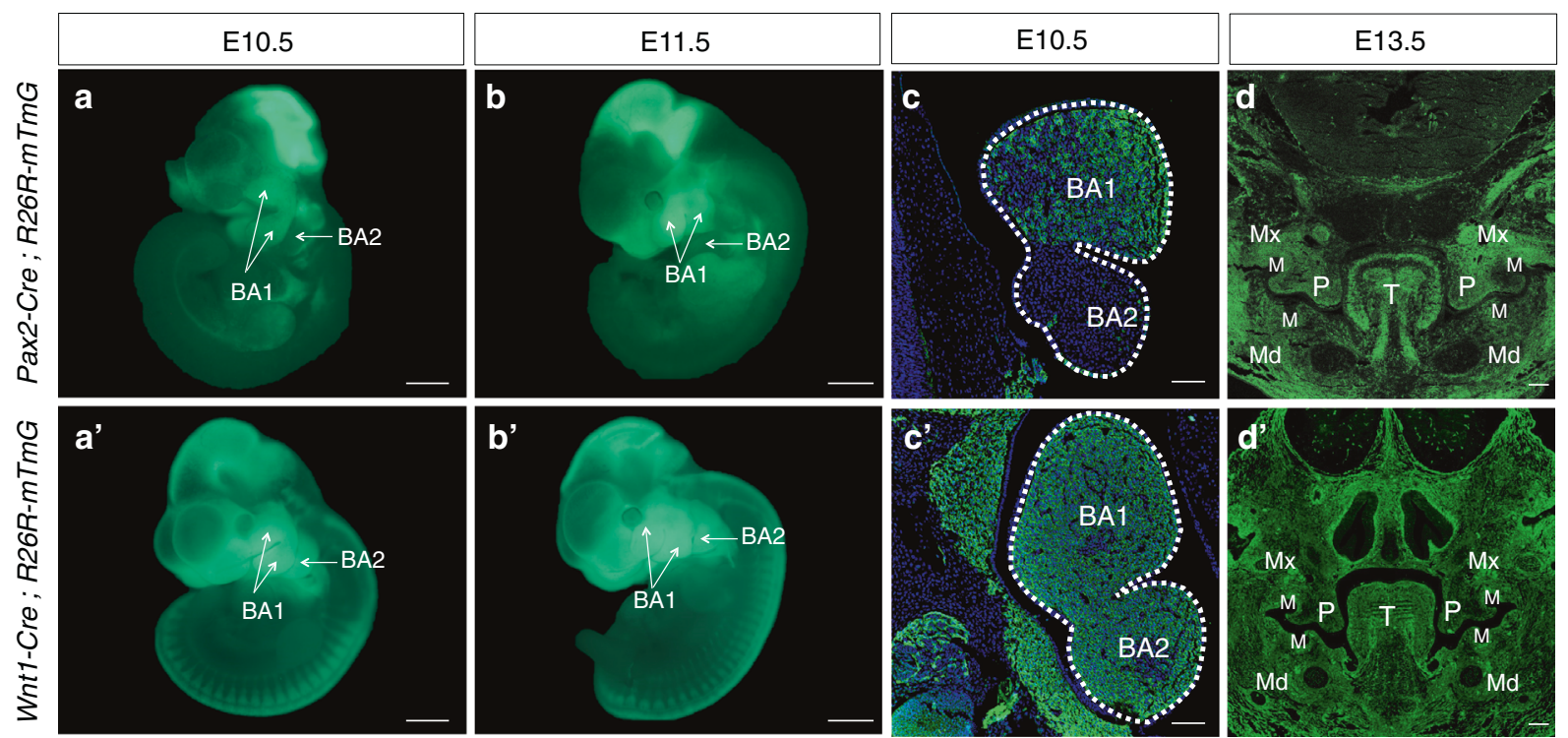

Fig. 1 The expression pattern of $\mathrm{Pax2}^{+}$and $\mathrm{Wnt}^{+}$cell lineages. a, $\mathbf{b}^{\prime} \mathrm{Pax2}^{+} \mathrm{CNC}$ cells, evidenced by GFP expression, were specifically detected in BA1 of Pax2-Cre; R26R-mTmG embryos at E10.5 (a) and E11.5 (b), as compared to the expression of GFP in both BA1 and BA2 of Wnt1-Cre;

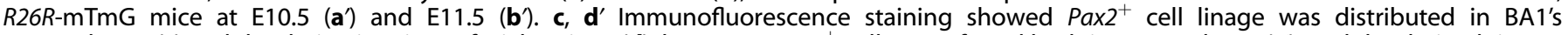
mesenchyme (c) and the derivatives in orofacial regions (d); however, Wnt $1^{+}$cells were found both in BA1 and BA2 (c'), and the derived tissues in craniofacial regions (d'). BA1 the first branchial arch, BA2 the second branchial arch, T, tongue; P, palatal shelf; Mx, Maxilla; Md, mandible; M, molar bud. Scale bars $=500 \mu \mathrm{m}\left(\mathbf{a}, \mathbf{b}^{\prime}\right)$; Scale bars $=100 \mu \mathrm{m}\left(\mathbf{c}, \mathbf{d}^{\prime}\right)$ 
Control
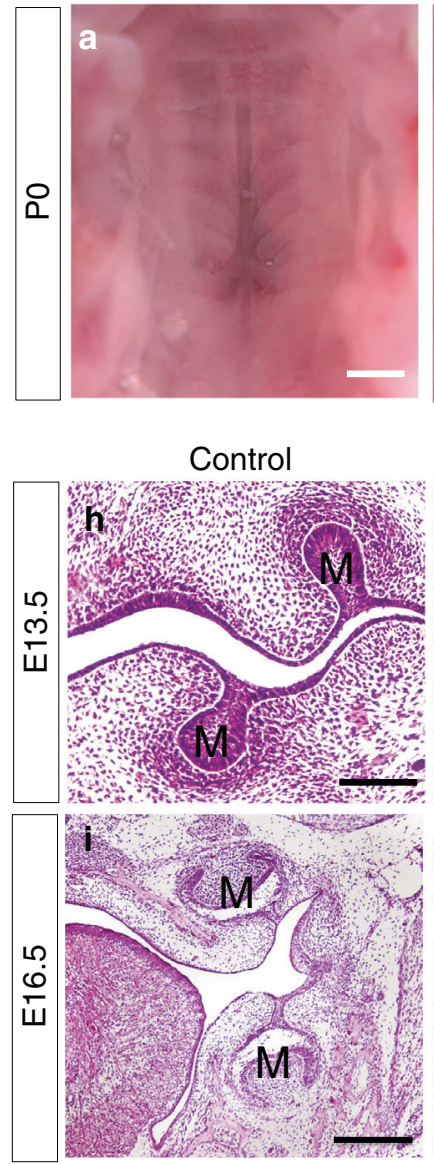

Pax2-Cre; pMes-Bmp4

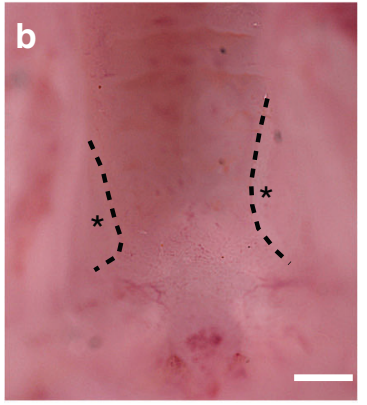

Pax2-Cre; pMes-Bmp4
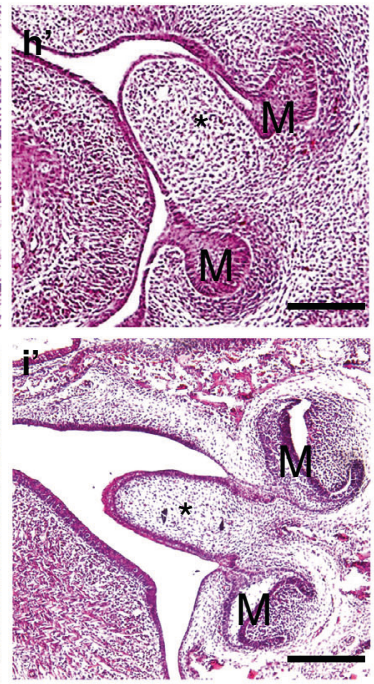

Control

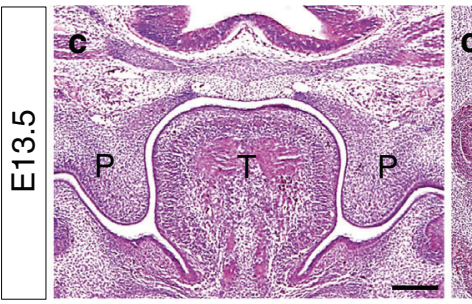

Pax2-Cre; pMes-Bmp4
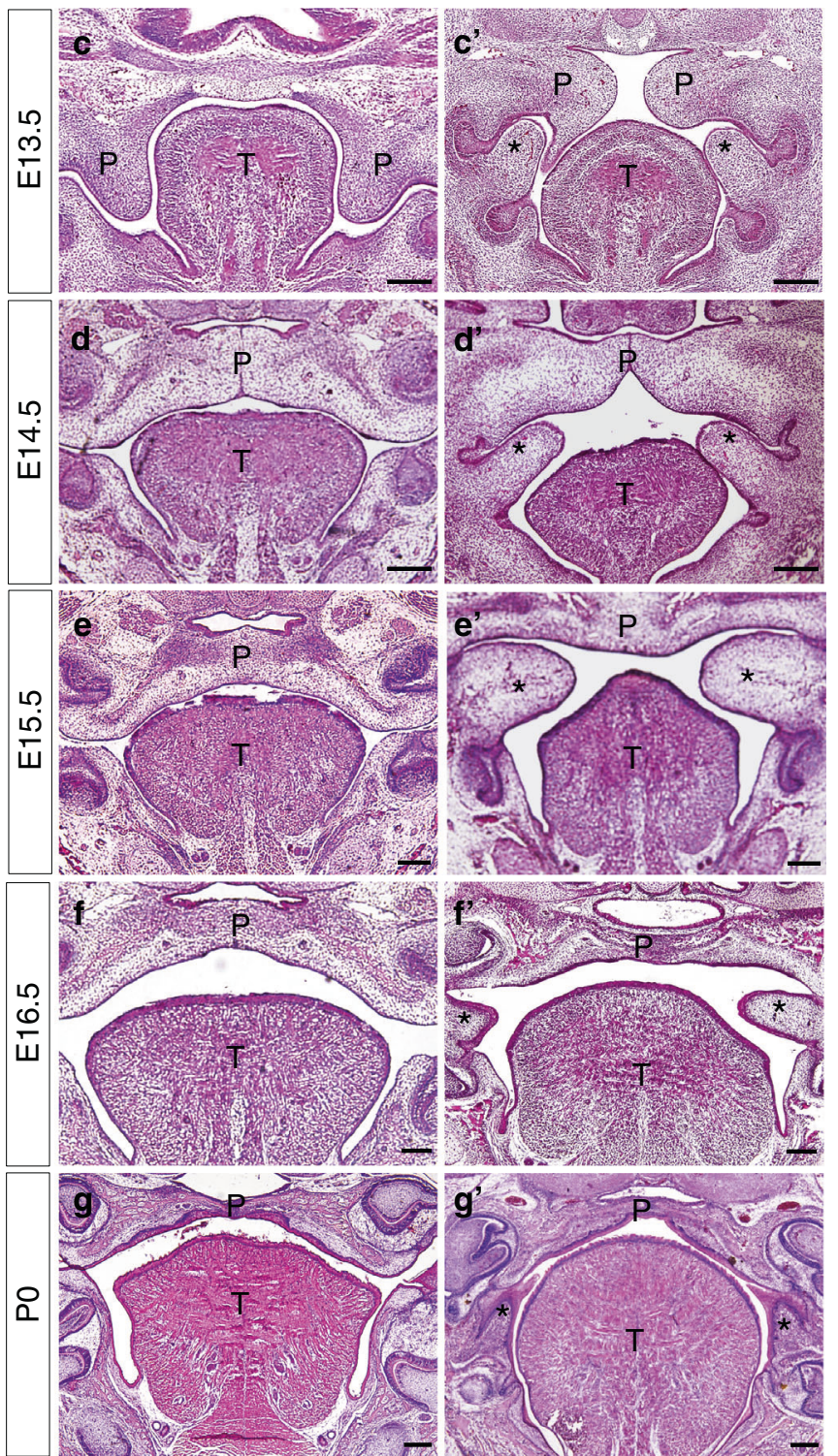

Fig. 2 Enhanced BMP4 signaling results in abnormal hyperplastic soft tissue in orofacial regions. $\mathbf{a}$, $\mathbf{b}$ Whole-mount view of palate in control (a) and Pax2-Cre; pMes-BMP4 mice (b) at P0. The mutant shows bilateral hyperplasia (b asterisks marked). $\mathbf{c}-\mathbf{g}^{\prime}$ Coronal sections of E13.5-P0 mice of controls' and mutants' heads reveal the abnormal bilateral hyperplastic soft tissues located between upper and lower molar buds $\left(\mathbf{c}^{\prime}-\mathbf{g}^{\prime}\right.$, asterisks marked). $\mathbf{h}, \mathbf{i}^{\prime}$ The growth direction of maxilla-mandibular molar buds was changed because of hyperplasia ( $\mathbf{h}^{\prime}, \mathbf{i}^{\prime}$ asterisks marked). T, tongue; P, palate; $M$, molar bud. Scale bars $=500 \mu \mathrm{m}\left(\mathbf{a}^{\prime}, \mathbf{b}^{\prime}\right)$; Scale bars $=100 \mu \mathrm{m}\left(\mathbf{c}-\mathbf{i}^{\prime}\right)$

morphology of the above organs showed barely difference between Pax2-Cre; pMes-BMP4 mice and controls (Fig. 3c). Notably, bony fusion between the upper and lower jaws specifically existed in Wnt1Cre; pMes-Bmp4 mice (Fig. 3b, green arrowheads). In control and Pax2-Cre; pMes-BMP4 mice, bilateral palatal shelves were successfully fused aside the tongue laterally at E16.5; however, cleft palate existed in Wnt1Cre; pMes-Bmp4 mice and the palatal shelves showed a reversed direction (the tip pointing dorsally to the nasal septum) (Fig. 3d-f). In Pax2-Cre; pMes-BMP4 mice, although there is no cleft palate, the abnormal hyperplastic soft tissues was found between maxilla-mandibular jaws (Fig. 3f, asterisks marked).

Overexpression of BMP4 signaling alters cellular behavior and gene expression in orofacial regions

To explore the properties of the hyperplastic soft tissue, we first detected the expression of Msx1, the critical gene of palate and teeth during development. In controls, Msx1 expressed in mesenchymal cells of tooth germ and anterior palate but not in middle and posterior parts of palatal shelf (Fig. 3a-c). In mutants, the expression level and pattern of Msx1 in palatal shelf were comparable to the controls; however, Msx1 expression in tooth buds slightly decreased and the $M s \times 1^{+}$cells were detected in mesenchyme of hyperplastic soft tissue from anterior to posterior part (Fig. 3a'-c). We also detect the osteogenic differentiation marker, Runx2, in orofacial regions of Pax2-Cre; pMes-BMP4 mice and the controls at E15.5. We found Runx2 expressed in the ossification areas of palate, maxilla, and mandible from anterior to posterior parts both in controls and mutants and the expression levels are comparable (Fig. $3 d-f$ ). However, Run $\times 2^{+}$cells were absent in the hyperplastic tissue (Fig. 3d'-f).

Because the hyperplastic soft tissue exists in the orofacial region of Pax2-Cre; pMes-BMP4 mice, we used Ki67 antibody to conduct a cell proliferation assay at E11.5 before the hyperplasia phenotype 
Control

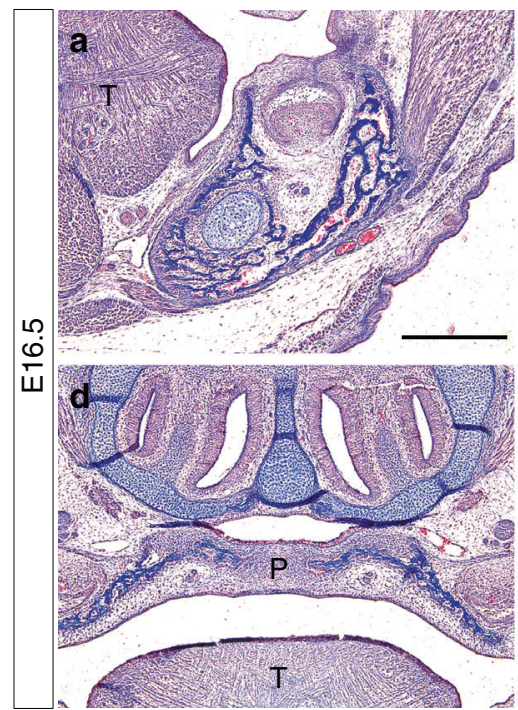

Wnt1-Cre; pMes-Bmp4

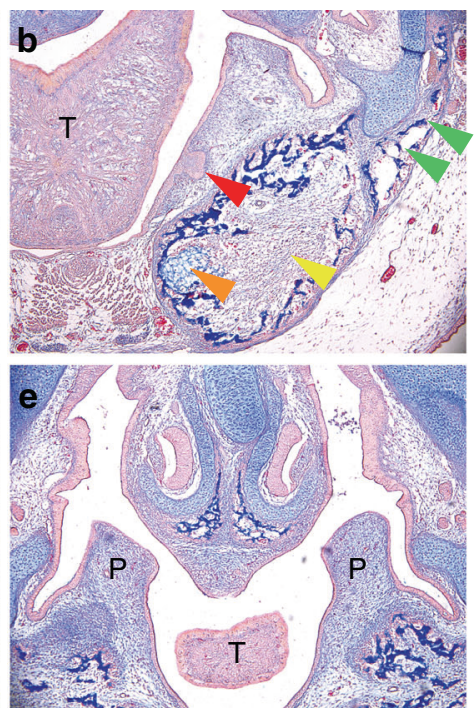

Pax2-Cre; pMes-Bmp4
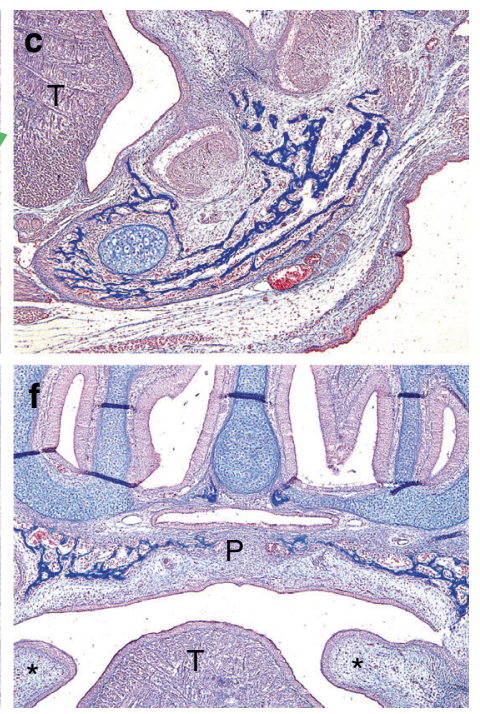

Fig. 3 Coronal sections revealed structures of molar buds, palate and mandible of control, Wnt1Cre; pMes-Bmp4 and Pax2-Cre; pMes-BMP4 head between the mandible and maxilla at E16.5. a-c The morphology of molar buds was distinctly defected in Wnt1Cre; pMes-Bmp4 mice (b, red arrowhead), but there is no obvious change between the Pax2-Cre; pMes-BMP4 (c) and control mice (a). Bony syngnathia was showed in Wnt1Cre; pMes-Bmp4 mice (b green arrowheads). The mandibular bony structures (yellow arrowhead) and Meckel's cartilage (orange arrowhead) were greatly inhibited in Wnt 1Cre; pMes-Bmp4 mice while these structures were comparable between Pax2-Cre; pMes-BMP4 (c) and control mice (a). d-f The palatal shelves were fused in control (d) and Pax2-Cre; pMes-BMP4 mice (f); however, cleft palate was showed in Wnt 1Cre; pMes-Bmp4 mice (e). Specifically, bilateral hyperplastic soft tissues (asterisks marked) were existed between maxilla-mandibular molar buds in Pax2-Cre; pMes-BMP4 mice. Scale bar $=500 \mu \mathrm{m}$

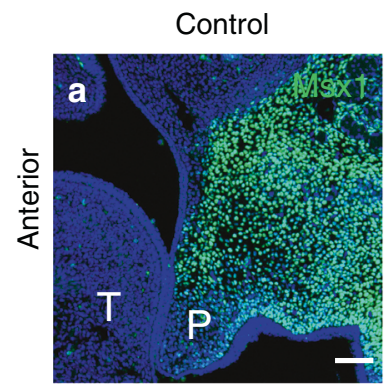

Pax2-Cre; pMes-Bmp4
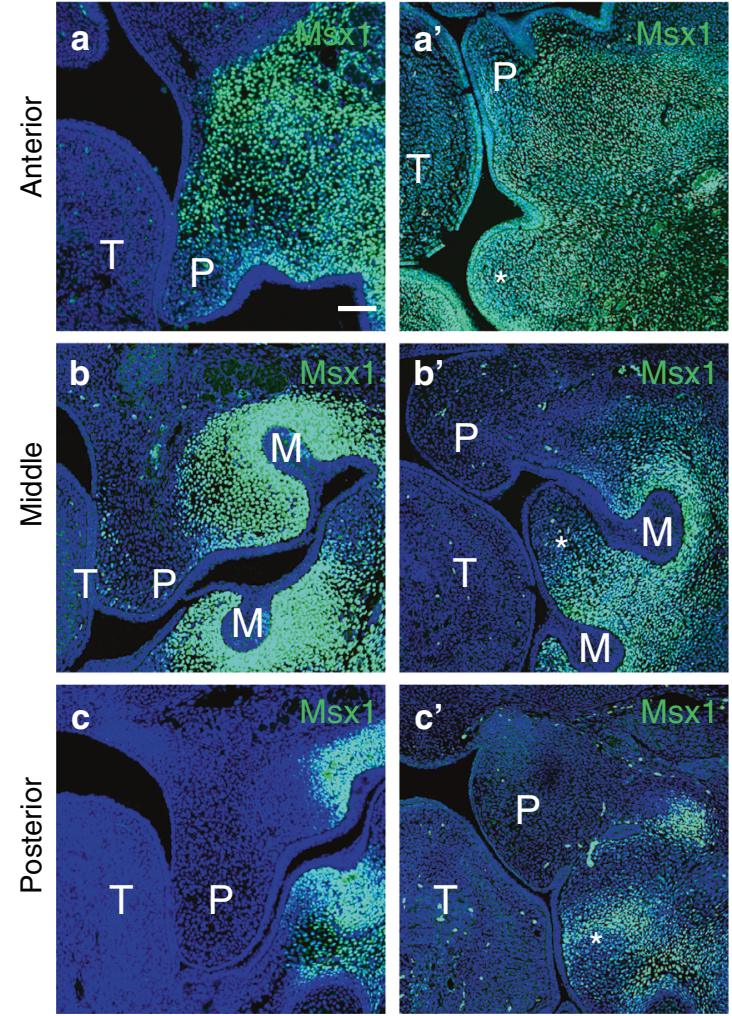
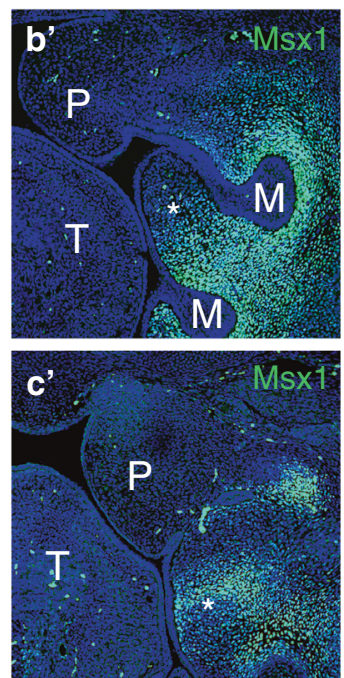

Control
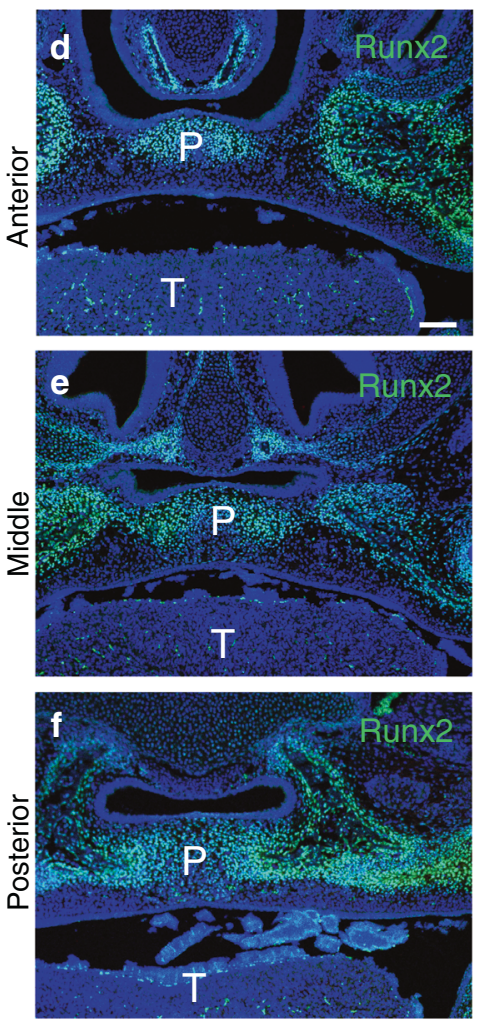

Pax2-Cre; pMes-Bmp4
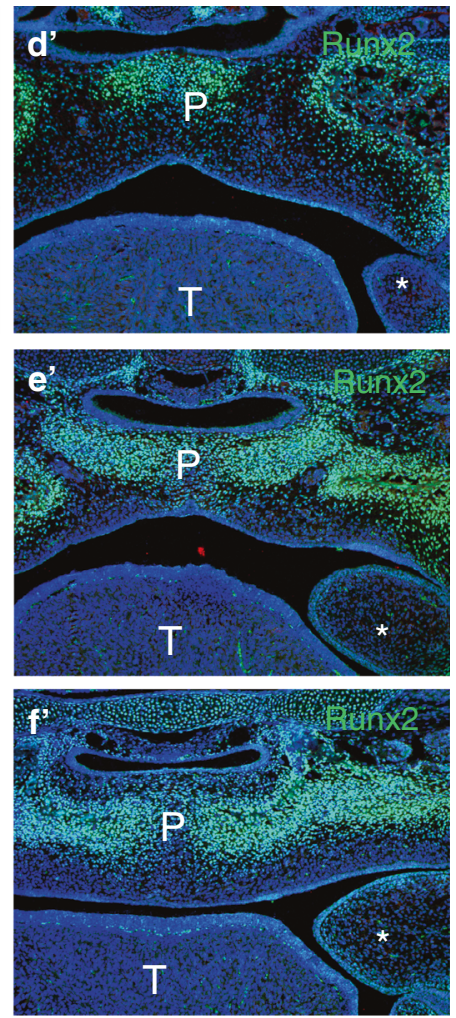

Fig. 4 Altered expression of orofacial landscape genes in Pax2-Cre; pMes-BMP4 mice. a-c' Immunostaining with anti-Msx1 antibody reveals the expression of Msx1 in anterior palatal shelves and mesenchyme of tooth buds in both controls and mutants (a, $\left.\mathbf{b}^{\prime}\right)$, and the mesenchymal cells of the abnormal hyperplasia ( $\mathbf{a}^{\prime}-\mathbf{c}^{\prime}$, asterisks marked). $\mathbf{d}-\mathbf{f}^{\prime}$ Runx2 were detected in ossification regions in maxilla-mandibular regions in

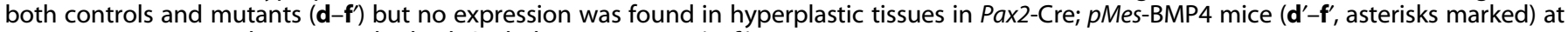
E15.5. T, tongue; $P$, palate; $M$, molar bud. Scale bars $=100 \mu \mathrm{m}\left(\mathbf{a}-\mathbf{f}^{\prime}\right)$ 
Control
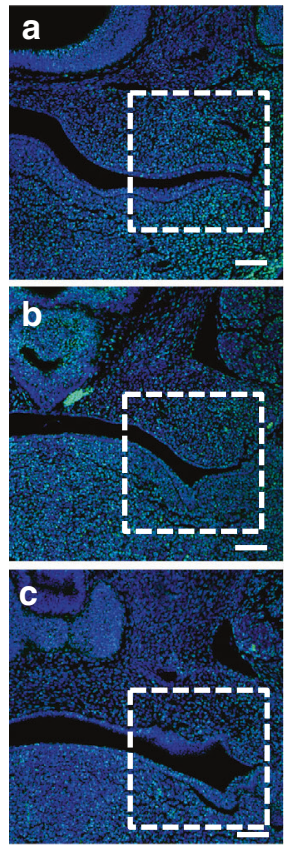
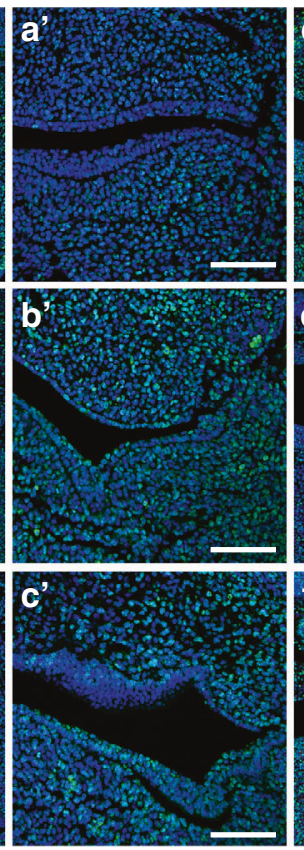

Pax2-Cre; pMes-Bmp4
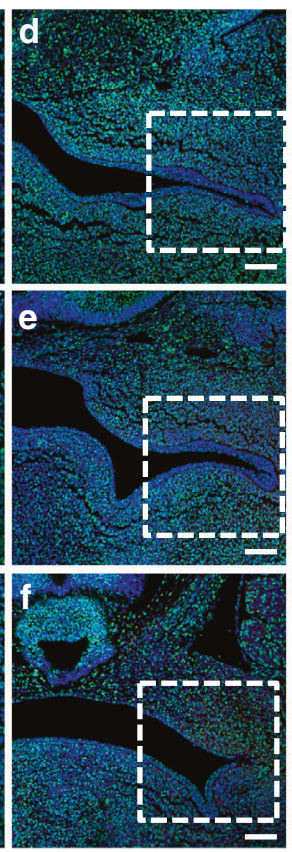
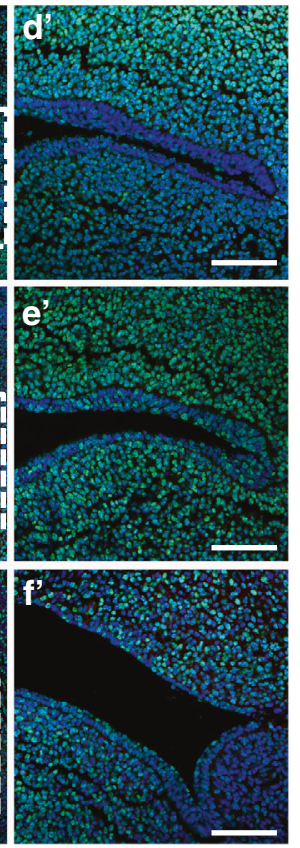
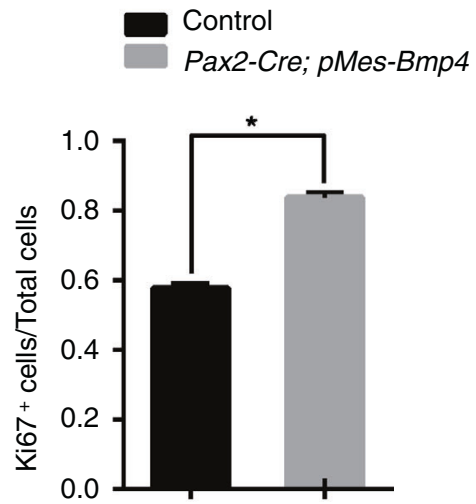

Ki67/DAPI

Fig. 5 Pax2-Cre-driven Bmp4 expression causes abundant cell proliferation in Pax2-Cre; pMes-BMP4 mice. Ki67-positive cells in three different maxilla-mandibular regions (a-f') reveal an abundant proliferation rate in Pax2-Cre; pMes-BMP4 mice compared to the control mice $\left(\mathbf{g}^{*} \mathrm{P}<0.05\right)$. T, tongue; $\mathrm{P}$, palate; $\mathrm{M}$, molar bud. Scale bars $=100 \mu \mathrm{m}\left(\mathbf{a}-\mathbf{f}^{\prime}\right)$

became recognizable. In the results, the $\mathrm{Ki} 67^{+}$cells were statistically significantly increased in Pax2-Cre; $p$ Mes-BMP4 mice (Fig. $4 ; P<0.05$ ). However, the levels of cell apoptosis in the mutants were comparable to the controls (Supplemental Fig. 2).

To explore the underlying mechanism of hyperplastic soft tissues, phosphorylated Smad1/5/8 (pSmad1/5/8) was also detected. There is no difference for expression of pSmad1/5/8 in palatal shelves between mutants and controls (Supplemental Fig. 3). However, pSmad1/5/8 specifically expressed in the basal part of the hyperplasia between upper and lower molar buds (Fig. 5b, arrowheads, Fig. 6).

\section{DISCUSSION}

Orofacial organs or tissues were originated from BA1-specific CNCs. In the field of orofacial development, researchers usually choose Cre transgenic mice to study the organogenesis or developing processes of the orofacial organs or tissues. However, there is no BA1-specific Cre mice currently. Therefore, to study the development of orofacial organs which are originated from $B A 1$, we better use an allele that has specific CNC cell labeling in BA1. In this study, we utilized Pax2-Cre; R26R-mTmG mice to trace the CNC-derived $\mathrm{Pax}^{+}$cells in the developing orofacial region and explore the specific effects of BMP4 signaling in these BA1 derivatives.

Pax2 is a transcriptional factor that is widely expressed during development in mammals. In the craniofacial region, Pax2 was found to appear in the developing otocyst and is considered as the marker of the otic placode ${ }^{39}$. Thus, Pax2-Cre mice were generated widely and used in the researches of inner ear development ${ }^{36,40}$. In our results, we surprisingly found Pax2 promoter drives expression in the neural crest post-migratory progenitors, resulting in permanent Cre-mediated GFP reporter activity in $\mathrm{BA} 1$ and there is barely $\mathrm{GFP}^{+}$cell in $\mathrm{BA} 2$. On the other hand, the $\mathrm{GFP}^{+}$cells existed both in BA1 and BA2 of Wnt1-Cre;
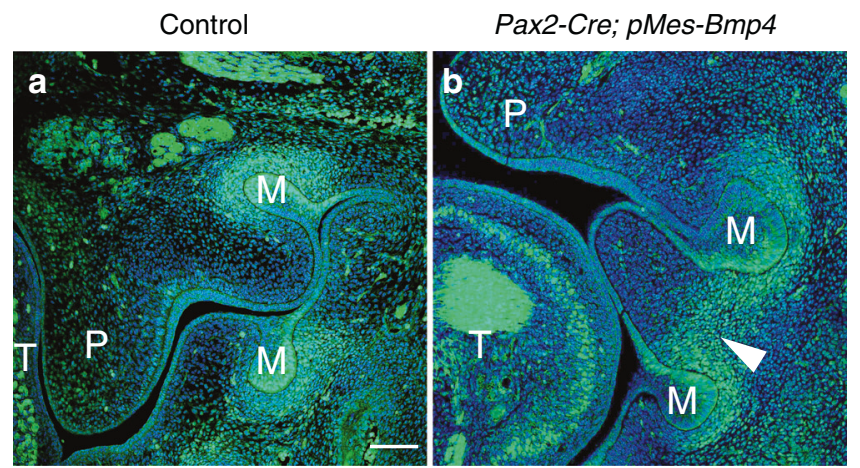

Fig. 6 The distributions of pSmad1/5/8 in the Pax2-Cre; pMes-BMP4 (b) and control mice (a) at E13.5. T, tongue; P, palate; M, molar bud. Scale bars $=100 \mu \mathrm{m}(\mathbf{a}, \mathbf{b})$

R26R-mTmG mice. Therefore, the $\mathrm{Pax}^{+}$cell lineage has a more specific expression pattern in CNC-derived BA1. The expression in skeletogenic mesenchyme of teeth, palate, upper and lower jaws, and tongue also confirmed that $\mathrm{Pax}^{+}$cell lineages contribute to BA1-derived orofacial organs during embryonic development in mice. One previous study using Pax2-Cre mice to conditionally knockout Dicer1 results in cleft palate ${ }^{41}$ supports the feasibility of Pax2-Cre allele in the field of orofacial organs' development.

It is acceptable that BMP4-mediated signaling is required for normal craniofacial development including teeth formation and palatogenesis ${ }^{21,42,43}$. Lack of Bmp4 in CNC cells results in abnormal size and morphology of teeth as well as some percentage of cleft palate ${ }^{21}$. On the other hand, over-activation of BMP4 signaling in CNC lineage by using Wnt1-Cre mice demonstrated a specific craniofacial abnormity, congenital bony 


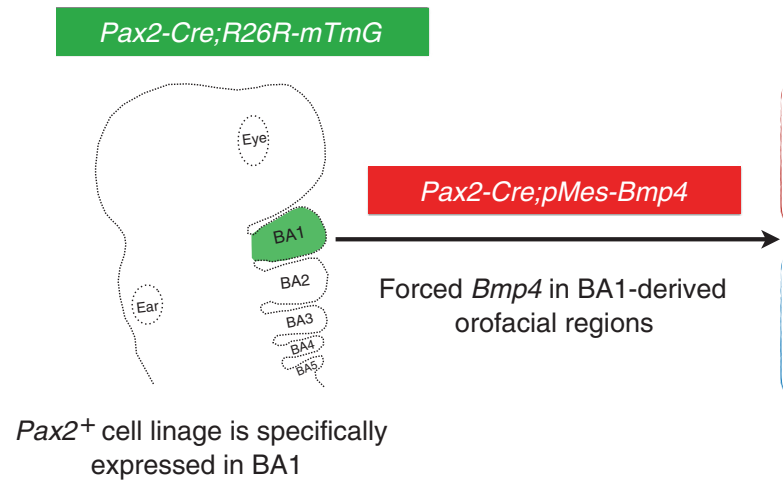

Orofacial Phenotypes

- Hyperplasia between upper and lower jaws

- Changed growth direction of tooth buds

- Significant cell proliferation

- Msx1 expressed in hyperplastic soft tissue

- Abnormal distribution of pSmad1/5/8

Altered cellular behaviors

Fig. 7 Schematic representations of specific cell lineage of Pax2-positive cells in BA1 as well as the effects of enhanced BMP4 signaling in $\mathrm{Pax}^{+}$lineage cells. BA the branchial arch

syngnathia, which manifested as bony fusion between maxilla and mandible, hypoplastic mandible, and cleft palate ${ }^{32}$. In our study, we investigated the effects of gain-of-function of BMP4 signaling by using BA1-specific Pax2-Cre transgenic mice. Surprisingly, the Pax2-Cre; pMes-BMP4 mice exhibit quite different orofacial phenotype, compared to the Wnt1-Cre; pMes-BMP4 mice. The severe defects of tooth, maxillamandibular bones, and complete cleft palate were shown in Wnt1-Cre; pMes-BMP4 mice; the results were comparable as previous study ${ }^{32}$. On the other hand, only the hyperplasia between upper and lower jaws was found in Pax2-Cre; pMesBMP4 mice in our study. It may suggest that the wide distribution of $\mathrm{Wnt}^{+}$cells leads to severe craniofacial phenotypes, but the BA1-derived $\mathrm{Pax2}^{+}$cell lineage has a limited distribution in orofacial regions and results in mild influences in orofacial organs.

To explore the influences on gene expression in orofacial regions and to confirm the properties of the hyperplastic tissue in Pax2-Cre; pMes-BMP4 mice, we detected the expression level of Msx1 and Runx2. Msx1 normally expresses in mesenchymal cells of tooth bud as well as anterior palate, but not in posterior palate $^{20}$. Here we found the expression levels of Msx 1 in anterior palate and teeth were comparable but Msx1 expresses in the mesenchyme of hyperplastic tissue from anterior to posterior parts in Pax2-Cre; pMes-BMP4 mice. Moreover, the hyperplastic tissue is located between upper and lower molar buds. It suggests this abnormal tissue is much closer to the tooth mesenchyme than palatal shelf. Hard tissues, including maxilla, mandible, hard palate, and the majority of teeth, are common but essential for morphogenesis and function in orofacial regions. Besides, in Wnt1-Cre; pMes-BMP4 mice, osteogenic differentiation was significantly inhibited ${ }^{32}$. So, we also detected the expression of Runx2 in Pax2-Cre; pMes-BMP4 mice. Runx2 expression in palate as well as the mesenchymal cells of tooth buds was comparable in mutants and controls; however, we cannot detect Runx2-positive cells in hyperplastic tissue of the mutant mice. This demonstrates that the hyperplasia does not fall into bony tissues.

Considering cell proliferation and apoptosis might be closely related to the phenotype of hyperplasia, we separately tested the rate of proliferation and apoptosis between mutants and controls. In our results, cell proliferation was significantly increased while cell apoptosis is comparable to the control group. It is consistent with the existence of hyperplastic soft tissue between upper and lower jaws in Pax2-Cre; pMes-BMP4 mice. However, these results are totally the opposite in Wnt1-Cre; pMes-BMP4 mice: there is no difference in cell proliferation rate between mutants and the control mice, but the abundant ectopic apoptosis in Wnt1-Cre; pMes-BMP4 mice. Therefore, cell behaviors driven by BMP4-related signaling might be decided by a different source of CNC cells and the hyperplasia is caused by abnormal abundance of cell proliferation.

In BMP4-mediated signaling, pSmad1/5/8 is an essential factor that can bind to Smad4, then enters the nucleus and interact with transcription factors to regulate downstream gene expression in orofacial organs such as palates and teeth ${ }^{13,21,28,44}$. Here, we showed a specific expression pattern of pSmad1/5/8 between upper and lower molar buds and the basal part of the hyperplastic soft tissue in Pax2-Cre; pMes-BMP4 mice. It suggests the hyperplasia might be associated with the abnormal activation of pSmad1/5/8 in mesenchymal cells between upper and lower molar buds. Based on our results, we summarize the specific cell lineage of $\mathrm{Pax}^{+}$and the effects of BMP4 signaling in Pax2-labeled BA1-derived mesenchymal cells in Fig. 7.

In conclusion, the present study indicates the importance of the Pax2-Cre mice to achieve specific neural crest cell labeling in BA1 and can be an optional tool for future studies during the development of orofacial organs. We also demonstrate the specific effects of BMP4 regulated signaling in BA1-derived mesenchyme compared to the Wnt1-Cre; pMes-BMP4 mice: enhanced BMP4 signaling leads to bilateral hyperplasia between upper and lower molar buds that is possibly caused by the significant increase of cell proliferation as well as the abnormal distribution of $\mathrm{pSmad} 1 / 5 / 8$ in the orofacial regions. Hence, our results further demonstrate the balanced BMP4 signaling is essential for the normal patterning and development in BA1derived orofacial organs.

\section{MATERIALS AND METHODS}

Animals

Pax2-Cre, R26R-mTmG, pMes-Bmp4, and pMes-Noggin transgenic mice used in this study have been previously described ${ }^{24,32,36,37}$. Staged Pax2-Cre; R26R-mTmG, Pax2-Cre; pMes-Bmp4 and Pax2-Cre; pMes-Noggin embryos or newborns were collected from timed pregnant females for further experiments. The usage of animals and the experiments were approved by the Institutional Animal Care and Use Committee of Fujian Normal University (Approval NO. IACUC-20200010).

Histological analysis

The embryonic heads from E13.5 through P0 were fixed in 4\% paraformaldehyde at $4{ }^{\circ} \mathrm{C}$ overnight. Then the samples were dehydrated in gradient alcohols. Subsequently, the samples were embedded with paraffin and sectioned according to the routine method. The $8 \mu \mathrm{m}$ slices were then subjected to hematoxylene and eosin (H\&E) staining or Azon red/Aniline blue staining as described ${ }^{32}$. 
Immunofluorescent staining

For immunofluorescent staining, the sliced samples were performed according to the manufacturer's instructions. The primary antibodies used were as follows: anti-GFP (Abcam, 1:1 000), anti-Ki67 (Abcam, 1:500), anti-Msx1 (Abcam, 1:500), antiRunx2 (Santa Cruz, 1:300), anti-pSmad1/5/8 (Millipore, 1:500), antiMF20 (Invitrogen, 1:200), type II Collagen (1:500, Abcam, ab34712), a-SMA (Invitrogen, 1:200). Alexa Fluor 488 (Thermo Fisher Scientific, 1:1 000) was used as secondary antibody. The tissues were then viewed under a confocal fluorescence microscope (Carl Zeiss, Göttingen, Germany). All the experiments in this study were repeated at least three times.

\section{TUNEL analysis}

The cell apoptosis was analyzed by using TUNEL assay following the use of the manufacturer's instruction of FragEL DNA Fragmentation Detection Kit, Fluorescein (Calbiochem, Darmstadt, Germany).

\section{Statistical analysis}

All the experiments and analyses were repeated at least three times. We randomly selected three different parts of maxillamandibular regions of the same size from at least three different samples and the number of the Ki67-positive $\left(\mathrm{Ki}_{67}{ }^{+}\right)$cells and apoptotic cells were counted by using ImageJ (version $1.46 \mathrm{r}$, National Institutes of Haealth) and were analyzed by the Student's $t$ test. ${ }^{*} P<0.05$ was regarded to be statistically significant. The results were presented as the means with standard deviation.

\section{ACKNOWLEDGEMENTS}

This work was supported by the National Natural Science Foundation of China (81900966) and the Key Research and Development Program of Sichuan Province (2020YFS0181) to J.X., the National Natural Science Foundation of China (82170918) to Z.H. We thank Dr. YiPing Chen of Tulane University and Dr. Yanding Zhang of Fujian Normal University for providing transgenic mice.

\section{AUTHOR CONTRIBUTIONS}

J.X. contributed to design, performed data analysis, and drafted the manuscript; M.C., Y.Y. and Q.Z. performed the experiments; M.S. and Z.H. contributed to design and conception and critically revised the manuscript.

\section{ADDITIONAL INFORMATION}

Supplementary information The online version contains supplementary material available at https://doi.org/10.1038/s41368-021-00142-4.

Competing interests: The authors declare no competing interests.

\section{REFERENCES}

1. Santagati, F. \& Rijli, F. M. Cranial neural crest and the building of the vertebrate head. Nat. Rev. Neurosci. 4, 806-818 (2003).

2. Minoux, M. \& Rijli, F. M. Molecular mechanisms of cranial neural crest cell migration and patterning in craniofacial development. Development 137, 2605-2621 (2010).

3. Cobourne, M. T. \& Sharpe, P. T. Tooth and jaw: molecular mechanisms of patterning in the first branchial arch. Arch. Oral. Biol. 48, 1-4 (2003).

4. Lee, S. H., Bedard, O., Buchtova, M., Fu, K. \& Richman, J. M. A new origin for the maxillary jaw. Dev. Biol. 276, 2070-2224 (2004)

5. Chai, Y. et al. Fate of the mammalian cranial neural crest during tooth and mandibular morphogenesis. Development 127, 1671-1679 (2000).

6. Bhattacherjee, V. et al. Neural crest and mesoderm lineage-dependent gene expression in orofacial development. Differentiation 75, 463-477 (2007).

7. Liu, $\mathrm{H}$. et al. Neural crest contribution to lingual mesenchyme, epithelium and developing taste papillae and taste buds. Dev. Biol. 368, 294-303 (2012).

8. Chen, G. et al. Specific and spatial labeling of P0-Cre versus Wnt1-Cre in cranial neural crest in early mouse embryos. Genesis 55, e23034 (2017).
9. Danielian, P. S., Muccino, D., Rowitch, D. H., Michael, S. K. \& McMahon, A. P. Modification of gene activity in mouse embryos in utero by a tamoxifeninducible form of Cre recombinase. Curr. Biol. 8, 1323-1326 (1998).

10. Minoux, M. et al. Gene bivalency at Polycomb domains regulates cranial neural crest positional identity. Science 355, 6332 (2017).

11. Kawakami, M., Umeda, M., Nakagata, N., Takeo, T. \& Yamamura, K. Novel migrating mouse neural crest cell assay system utilizing P0-Cre/EGFP fluorescent time-lapse imaging. BMC Dev. Biol. 11 (2011).

12. Lan, Y., Wang, Q., Ovitt, C. E. \& Jiang, R. A unique mouse strain expressing Cre recombinase for tissue-specifc analysis of gene function in palate and kidney development. Genesis 45, 618-624 (2007).

13. Li, N. et al. Altered BMP-Smad4 signaling causes complete cleft palate by disturbing osteogenesis in palatal mesenchyme. J. Mol. Histol. https://doi.org/ 10.1007/s10735-020-09922-4 (2020).

14. Lu, Y. et al. DMP1-targeted Cre expression in odontoblasts and osteocytes. J. Dent. Res. 86, 320-325 (2007).

15. Xu, J., Wang, L., Huang, Z., Chen, Y. \& Shao, M. Exogenous FGF8 signaling in osteocytes leads to mandibular hypoplasia in mice. Oral. Dis. 26, 590-596 (2020)

16. Maes, C. et al. Osteoblast precursors, but not mature osteoblasts, move into developing and fractured bones along with invading blood vessels. Dev. Cell. 19, 329-344 (2010).

17. Guo, F. et al. Bmp2 deletion causes an amelogenesis imperfecta phenotype via regulating enamel gene expression. J. Cell. Physiol. 230, 1871-1882 (2015).

18. Kishigami, S. et al. BMP signaling through ACVRI is required for left-right patterning in the early mouse embryo. Dev. Biol. 276, 185-193 (2004).

19. Graf, D., Malik, Z., Hayano, S. \& Mishina, Y. Common mechanisms in development and disease: BMP signaling in craniofacial development. Cytokine Growth F. R. 27 129-139 (2016).

20. Zhang, Z. et al. Rescue of cleft palate in Msx1-deficient mice by transgenic Bmp4 reveals a network of BMP and Shh signaling in the regulation of mammalian palatogenesis. Development 129, 4135-4146 (2002).

21. Jia, S. et al. Roles of Bmp4 during tooth morphogenesis and sequential tooth formation. Development 140, 423-432 (2013).

22. Chen, Y., Wang, Z., Chen, Y. \& Zhang, Y. Conditional deletion of Bmp2 in cranial neural crest cells recapitulates Pierre Robin sequence in mice. Cell. Tissue Res. 376, 199-210 (2019).

23. Li, L. et al. Augmented BMPRIA-mediated BMP signaling in cranial neural crest lineage leads to cleft palate formation and delayed tooth differentiation. PLoS ONE 8, e66107 (2013).

24. Xiong, W. et al. Hand2 is required in the epithelium for palatogenesis in mice Dev. Biol. 330, 131-141 (2009).

25. Huang, X. F. \& Chai, Y. Molecular regulatory mechanism of tooth root development. Int. J. Oral. Sci. 4, 177-181 (2012).

26. $\mathrm{Hu}, \mathrm{X}$. et al. Noggin is required for early development of murine upper incisors. J. Dent. Res. 91, 394-400 (2012).

27. Wang, Y. et al. BMP activity is required for tooth development from the lamina to bud stage. J. Dent. Res. 91, 690-695 (2012).

28. Yuan, G. et al. The non-canonical BMP and Wnt/ $\beta$-catenin signaling pathways orchestrate early tooth development. Development 142, 128-139 (2015).

29. Komatsu, Y. et al. Augmentation of Smaddependent BMP signaling in neural crest cells causes craniosynostosis in mice. J. Bone Miner. Res. 28, 1422-1433 (2013).

30. Wang, Y., Zheng, Y., Chen, D. \& Chen, Y. Enhanced BMP signaling prevents degeneration and leads to endochondral ossification of Meckel's cartilage in mice. Dev. Biol. 381, 301-311 (2013).

31. Bonilla-Claudio, $M$. et al. Bmp signaling regulates a dose-dependent transcriptional program to control facial skeletal development. Development 139, 709-719 (2012).

32. He, F. et al. Directed Bmp4 expression in neural crest cells generates a genetic model for the rare human bony syngnathia birth defect. Dev. Biol. 391, 170-181 (2014).

33. Gu, S. et al. BMPRIA mediated signaling is essential for temporomandibular joint development in mice. PLOS ONE 9, e101000 (2014).

34. Shirakura, M. et al. Extracellular matrix mediates BMP-2 in a model of temporomandibular joint osteoarthritis. Cells Tissues Organs 204, 84-92 (2017).

35. He, F. et al. Modulation of BMP signaling by Noggin is required for the maintenance of palatal epithelial integrity during palatogenesis. Dev. Biol. 347 109-121 (2010).

36. Ohyama, T. \& Groves, A. K. Generation of Pax2-Cre mice by modification of a Pax2 bacterial artificial chromosome. Genesis 38, 195-199 (2004).

37. Muzumdar, M. D., Tasic, B., Miyamichi, K., Li, L. \& Luo, L. A global doublefluorescent Cre reporter mouse. Genesis 45, 593-605 (2007).

38. Arnold, J. S. et al. Tissue-specific roles of Tbx1 in the development of the outer, middle and inner ear, defective in 22q11DS patients. Hum. Mol. Genet. 15, 1629-1639 (2006). 
39. Pfeffer, P. L. et al. The activation and maintenance of Pax2 expression at the midhindbrain boundary is controlled by separate enhancers. Development 129, 307-318 (2002).

40. Ohyama, T. Unraveling inner ear induction by gene manipulation using Pax2-Cre BAC transgenic mice. Brain Res. 1277, 84-89 (2009).

41. Barritt, L. C. et al. Conditional deletion of the human ortholog gene Dicer1 in Pax2Cre expression domain impairs orofacial development. Indian. J. Hum. Genet. 18, 310-319 (2012).

42. Bei, M., Kratochwil, K. \& Maas, R. L. BMP4 rescues a non-cellautonomous function of Msx1 in tooth development. Development 127, 4711-4718 (2000).

43. Zhao, X. et al. Transgenically ectopic expression of Bmp4 to the Msx1 mutant dental mesenchyme restores downstream gene expression but represses Shh and Bmp2 in the enamel knot of wild type tooth germ. Mech. Dev. 99, 29-38 (2000).

44. Yuan, G., Zhan, Y., Gou, X., Chen, Y. \& Yang, G. TGF- $\beta$ signaling inhibits canonical BMP signaling pathway during palate development. Cell Tissue Res. 371, 283-291 (2018). (c) Open Access This article is licensed under a Creative Commons Attribution 4.0 International License, which permits use, sharing, adaptation, distribution and reproduction in any medium or format, as long as you give appropriate credit to the original author(s) and the source, provide a link to the Creative Commons license, and indicate if changes were made. The images or other third party material in this article are included in the article's Creative Commons license, unless indicated otherwise in a credit line to the material. If material is not included in the article's Creative Commons license and your intended use is not permitted by statutory regulation or exceeds the permitted use, you will need to obtain permission directly from the copyright holder. To view a copy of this license, visit http://creativecommons. org/licenses/by/4.0/.

(c) The Author(s) 2021 